\title{
Courrier concernant la grève de la faim et l'alimentation forcée
}

\section{Courrier du Comité international de la Croix-Rouge CICR au président de la FMH}

Monsieur le Président, Cher Confrère,

Nous avons lu avec attention dans la presse votre récente prise de position concernant le rôle et le devoir des médecins dans les grèves de la faim en détention.

Nous vous confirmons que la position du Comité international de la Croix-Rouge (CICR) rejoint la vôtre. Le CICR se réfère en la matière à la Déclaration de Malte de l'Association Médicale Mondiale qui, tout en reconnaissant les dilemmes éthiques posés par ces situations, rappelle que le respect de l'autonomie du patient est une exigence éthique fondamen-

\section{Courrier au Bulletin des médecins suisses}

In der NZZ vom 7.9.2010 warf der Generalsekretär der Schweizerischen Akademie der Medizinischen Wissenschaften SAMW unter dem Titel «Hungerstreik als politisches Problem» dem Bundesgericht wegen dessen Entscheid, dass Ärzte eine Zwangsernährung «auf Anordnung» auszuführen haben, vor, seine Kompetenz überschritten zu haben. Das Gericht habe sich über das Gebot der ärztlichen Standesethik, die Autonomie des Patienten zu respektieren, hinweggesetzt. Der Gesetzgeber sei gefordert, die Rechte der Strafgefangenen zu erweitern. Prof. M. Müller vom Institut für öffentliches Recht der Universität Bern ortet in der NZZ vom 9.9.2010 «in der Ärzteschaft ein grundlegendes Missverständnis über die Rolle und Funktion der Gefängnisärzte». Die Vornahme einer Massnahme, die gegen die ärztliche Standesethik verstösst, gelte wohl für Privatärzte, nicht aber für Gefängnisärzte, die Amtspersonen sind. Diese seien Teil der Gefängnisverwaltung und unterstünden der administrativen Befehlsgewalt. Obwohl dem Gefängnisarzt von seiner Gefängnisleitung weitestgehend fachliche Autonomie zugestanden werde, könne es dazu kommen, dass die Strafvollzugsbehörde von ihm die Durchführung einer medizinischen Massnahme verlangt, die seinem persönlichen Berufsethos oder gar den Standesregeln widerspricht. Die Strafvollzugsbehörde habe das staatliche Recht in jedem Fall durchzusetzen.

Die von mir in einem Leserbrief begründete Unterstützung des Standpunktes der SAMW wurde von der NZZ nicht publiziert. Meine Bedenken gegen die Politisierung des verfassungsmässig geschützten Rechts auf persönliche Freiheit lauten wie folgt: Mit der Anordnung, dass Ärzte eine Zwangsernährung eines als tale. De ce fait l'alimentation forcée d'un détenu n'est pas acceptable. De même, comme vous le soulignez bien, le respect de la liberté thérapeutique du médecin et celui de la confidentialité médicale, sont des conditions fondamentales de l'exercice de la médecine.

A cet égard le CICR s'inquiète des risques d'une érosion de ces principes de l'éthique médicale, et des graves conséquences pour les personnes privées de liberté.

Dr Paul Bouvier, Conseiller médical du CICR, Genève

urteilsfähig beurteilten Strafgefangenen, der die Nahrungsaufnahme verweigert, durchzuführen haben, setzt sich dieser Bundesgerichtsentscheid nicht nur über das Gebot der ärztlichen Standesethik, die Autonomie des Patienten zu respektieren, hinweg. Der Gerichtsentscheid missachtet das verfassungsmässig geschützte Recht auf persönliche Freiheit. Dieses Recht bedeutet [1], dass ein Arzt ohne Einwilligung des urteilsfähigen, angemessen über Eingriff und Risiken aufgeklärten Patienten («informed consent») keinerlei Befugnis zur Untersuchung und zu therapeutischen Massnahmen hat. Das Verbot, Eingriffe ohne Einwilligung des urteilsfähigen Patienten durchzuführen, wird im Art. 28a des Zivilgesetzbuches ZGB in dem Sinne verstärkt, als ein nicht gerechtfertigter Eingriff mit Sanktionen (Schadensersatz, Verbot, Genugtuung) ausgestattet wird.

Die bundesgerichtliche Anordnung zur Zwangsernährung des Walliser Hanfbauern Rappaz missachtet somit nicht nur das Gebot der ärztlichen Standesethik, sondern auch das verfassungsmässig geschützte Recht auf persönliche Freiheit, das die Ärzte respektieren müssen. Würde Rappaz als urteilsunfähig beurteilt, wären die Ärzte zur Hilfe in Form einer Ernährung des urteilsunfähigen Patienten verpflichtet [2], selbst dann, wenn er sich gegen die in seinem Interesse liegende Behandlung wehren würde. Die Diskussion ist eröffnet. Gilt das Privatrecht im Arzt-Patienten-Verhältnis für Strafgefangene nicht? Ist die Politisierung des verfassungsmässig geschützten Rechts auf persönliche Freiheit richtig oder nicht akzeptabel?

Prof. Dr. med. Max Geiser, Wabern 